\title{
Aspects of Train Systems Simulation
}

\author{
Martin R. Kuhn ${ }^{1}$ Yang Ji ${ }^{2}$ Bo Wang ${ }^{2}$ Xiang $\mathrm{Li}^{2}$ Bohui Liu ${ }^{2}$ Feng Sha ${ }^{2}$ Dunwen Gan ${ }^{3}$ Feng Gao \\ ${ }^{1}$ Yuanda SimTek GmbH, Germany, martin.kuhn@yuandasimtek.de \\ ${ }^{2}$ SimTek CO, China, \{yang.ji, bo.wang, li.xiang, bohui.liu, feng.sha \\ acnydsimtek.com \\ ${ }^{3}$ BEIJING ZONGHENG ELECTRO-MECHANICAL TECHNOLOGY DEVELOPMENT CO, \\ \{gandunwen, gaofeng\} @ zemt.cn
}

\begin{abstract}
This paper present needs and implementations for system modeling of high speed trains with focus on the Beijing-Zhangjiakou Intercity Railway. Different scenarios are proposed which are relevant in systems design. The implementation with Modelica is discussed and demonstrated for the rail-wheel contact and mechanical, logical, electrical and thermal systems.
\end{abstract}

Keywords: High speed train, systems, electrical, mechanical, thermal, rail-wheel contact, single phase, traction

\section{Motivation}

\subsection{The Chinese high speed trains system}

Chinese Railway High-speed (CRH) was first introduced in 2007 and was developed further with operating speeds of $250-300 \mathrm{~km} / \mathrm{h}$. The train system was able to significantly reduce the further growth of air traffic. Even for long distances as Beijing-Shanghai with $1077 \mathrm{~km}$ the travelers are attracted by scheduled travel times of 288 minutes in relation to 135 minutes by airplane. Assuming 60 minutes additional journey time for the train respectively 150 minutes to the outlying airports the ratio shrinks to 348 minutes to 285 . In addition the train service is operated roughly every 5 minutes with prices around $85 \%$ of the air ticket.

The Chinese high speed train program follows the principle of Electric Multiple Units (EMU), where the train consists of self-propelled carriages with no separate locomotive and traction motors incorporated within a number of the carriages. The same concept applies for example for the French AGV, Italian Pendolino, Japanese Shinkansen or German ICE 3. Beijing-Zhangiiakou Intercity railway, as a supporting project of the Beijing 2022 Olympic and Paralympic Winter Games, is the first high-speed train with an operational speed of $350 \mathrm{~km} / \mathrm{h}$ and automatic driving. It will operate in an alpine and windy region where it will reduce the commuting time between Beijing and Zhangjiakou to an hour. Assembly of the EMUs is planned by end of 2018 and the adaption and test verification shall be completed in the first half of 2019.
ZEMT is one of the core partners in the new development. The company identified a further need for model-based system design, optimization, fault analysis and diagnosis. Train systems simulation is a well known application of Modelica (Belmon and Liu, 2011; Dumont and Maurer, 2012; Franke and Wiesmann, 2014; Heckmann et al., 2014; Frilli et al., 2016). Therefore, SimTek has been commissioned to develop a Modelica-based train systems library for further virtual testing and verification of the BeijingZhangjiakou high-speed train. This paper presents typical scenarios which are relevant in systems design. The implementation of the library with Modelica is discussed and demonstrated for the rail-wheel contact and mechanical, logical, electrical and thermal systems, with special attention to the rail-wheel contact and electrical power off-take. The models are partly generic and do not necessarily reflect the real system layout.

\subsection{Applications for simulation}

The following list provides typical applications for simulation based studies:

- Vehicle energy consumption estimation for systems and supply network optimization

$\circ$ Estimation of the energy consumption of all subsystems

- Energy consumption of air-conditioning and auxiliary system

- Energy consumption in different climate and weather environments and roads

- Electric grid harmonic estimation for topology and filter selection

- Estimate the harmonics according to industrial standards

- Traction system thermal capacity estimation for cooling system layout and control of power reduction

- Cooling models

- Simplification and parameter estimation of fluid models involving heat exchange

- Simplification and parameter estimation of models of the detailed electric power switching with thermal effect 
- Simplification and parameter estimation of models of the coupled power electrics and dynamic thermal model

- Driven cars stability estimation

- Lateral dynamics analysis

- Vertical dynamics analysis

\section{Modeling}

\subsection{Overview on libraries}

The modeling for the ZEMT Project of the virtual EMU is structured into four libraries, which will be commercialized. Their layout is shown in Figure 1.

This paper is focused on the unique feature of train modeling with the introduction of the mechanical modeling and thermalfluid modeling in the TrainDynamics Library, and the control logics modeling and electrical traction modeling in TractionSystemLibrary.

The TrainDynamicsLibrary supports the following models:

- Dynamic multi-body models of coach body and bogie

- Dynamic wheel/rail contact model.

- Thermal management, simple air conditioning and cooling unit of traction system, including motor cooling unit, converter cooling unit and transformer cooling unit.

- Water management, i.e., water supply and drainage system

- $\quad$ Passenger model, including weight, temperature, $\mathrm{O}_{2}$ consumption, water consumption

- Environment model, including temperature, pressure, humidity and wind resistance

- $\quad$ Path model, including 2 kinds of $x-y-z$ coordinates and $\mathrm{x}-\mathrm{z}-\mathrm{R}$ ( bend road).

The usage and contents of the TrainDynamicsLibrary:

- Analyze the stability of the train during hunting movement,

- Analyze the stability of the train while passing the winding railway line,

- Estimation and analysis of the energy consumption of the train.

ElectricTractionLibrary provide the dynamic models of electric traction system and control system. Subsystems include:

- Communication models,

- Signal system models,

- networks models,

- System integration and model in loop.

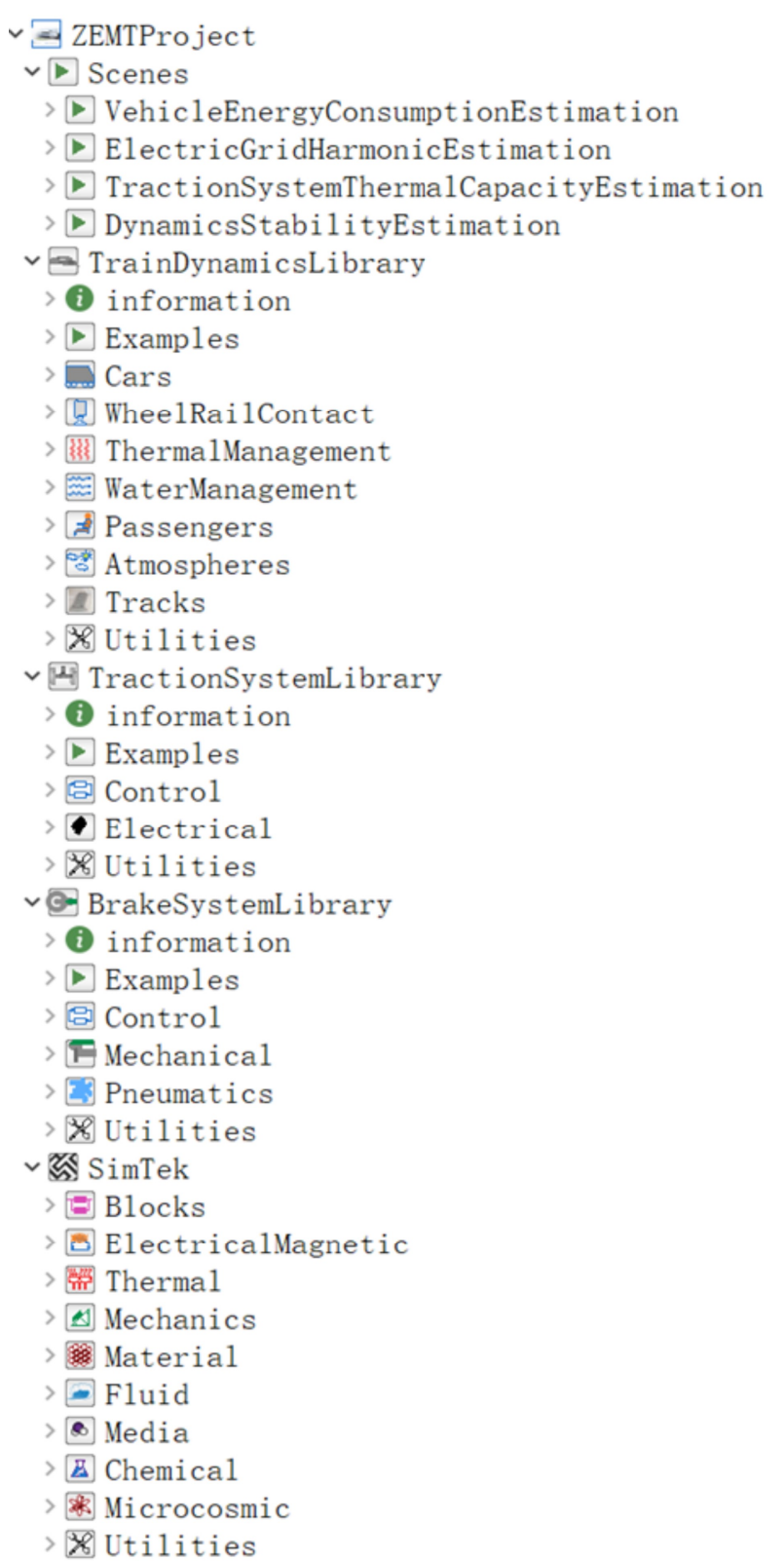

Figure 1. ZEMT project and SimTek commercial libraries.

\subsection{Train Dynamics Modeling}

\subsubsection{Implementation}

The mechanical structure of the EMU can be modeled by the simplified five elements model whose topology is shown in Figure 2. It includes:

- Coach body

- Bogie

- Wheel-sets

- The secondary suspension

- air spring model

- anti-roll var model

- vertical damper

- lateral damper

- yaw damper

- The primary suspension 
- spring damper.

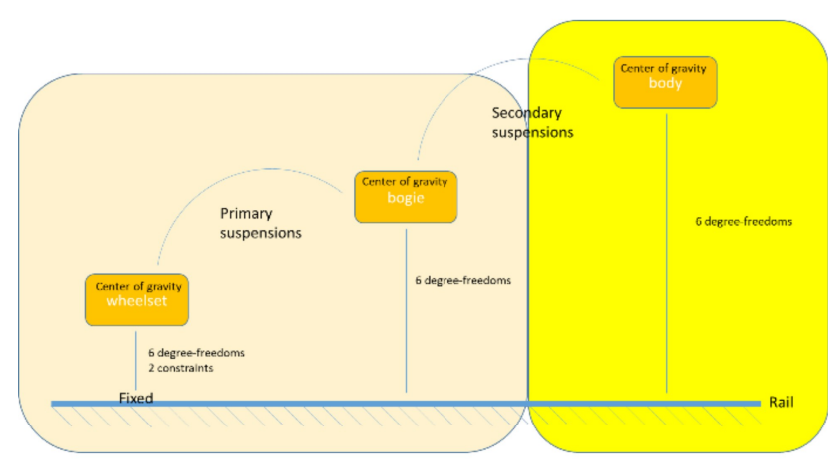

Figure 2. Topology structure about mechanical model.

All the design parameters and the characteristic curve of the suspension are based on identified test data. For example, the lateral damper characteristic is shown in Figure 3.

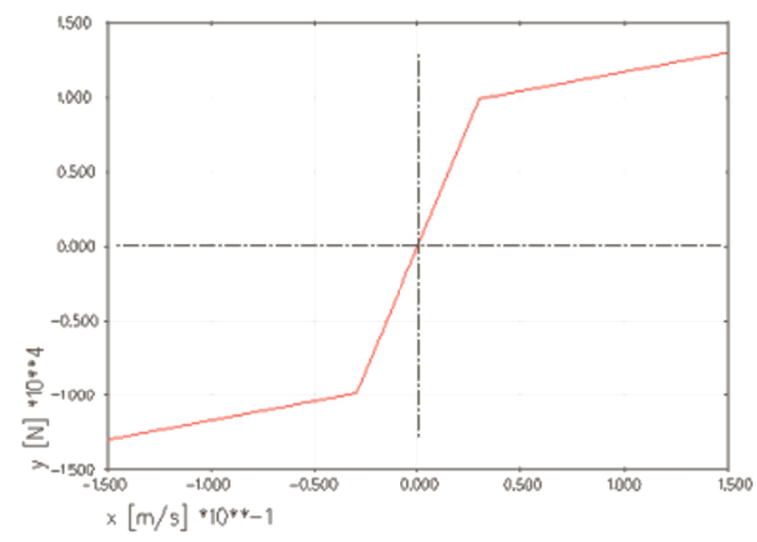

Figure 3. Lateral damper characteristic map.

The contact model is the most difficult part of the vehicle dynamics analysis. For automobiles, it's the adhesion forces to ensure the continuous motion. For aircraft, it's the air forces due to the contact between wing and air to guarantee the flight. The forces along motion direction and the perpendicular direction should be considered in the contact model. In a small range of the contact angle, the friction forces $F$ in lateral direction is linear to the contact angle $\alpha$, and its
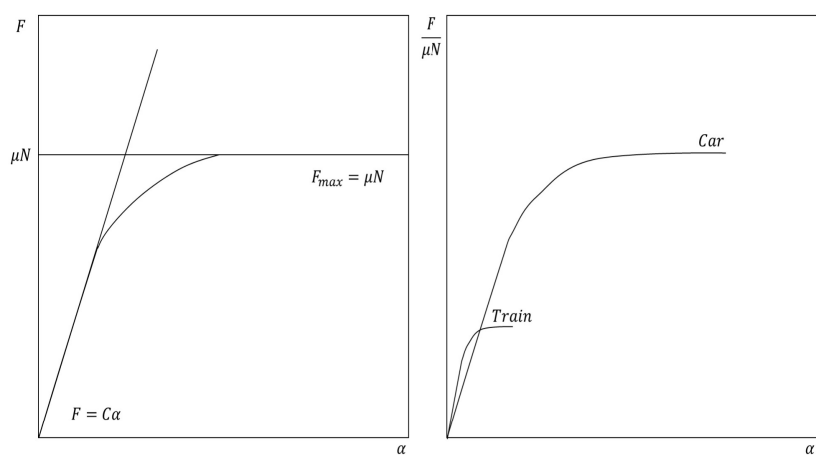

Figure 4. Left: Friction force in relation to contact angle. Right: Difference between trains and automobiles contact model. maximum is linear to the vertical load (Masao, 2008). This is shown in the left of Figure 4.

As for the trains, the linearity range of the contact angle is smaller and the curvature is more complex than for cars, as shown in the right of Figure 4.

For the EMU study, the integrated train energy consumption needs to be analyzed and controlled in real-time, where the simulation speed is more critical than the accuracy. In (Heckmann et al., 2014) an algorithm for fast calculation of wheel-rail forces with Modelica was proposed. Here, an alternative very fast approach is demonstrated. The local coordinates of the model are depicted in Figure 5 (Masao, 2008). In the six degrees of freedom of the train wheel set, the motion along $\mathrm{z}$ axis and rotation around $\mathrm{x}$ axis are constrained by the rail. Only $2 \mathrm{D}$ planar motion is allowed, which is the forward/backward movement in $\mathrm{x}$ direction, left/right movement in $\mathrm{y}$ direction, rotation around $\mathrm{z}$ direction $(\psi)$ and 1D rotation around $\mathrm{y}$ axis $(\theta)$. Considering the suspension of the train, the forces applied on the wheels by the train body are assumed to be one-dimensional in direction of $\mathrm{z}$ axis. This means a combination of 2D and 1D model was built, in use of the PlanarMechanics library (Zimmer, 2012).

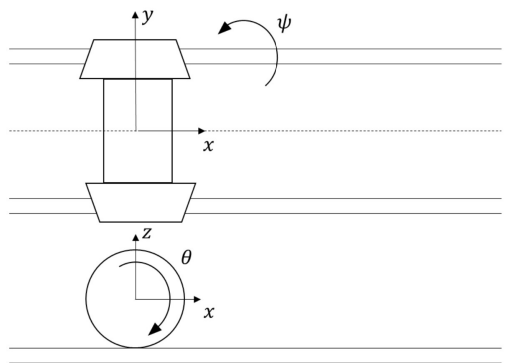

Figure 5. The moving coordinate system of the contact model.

The transformation between the local $(\mathrm{x}, \mathrm{y}, \psi)$ and global reference system $(\mathrm{X}, \mathrm{Y}, \psi)$ is performed by equations (1) and (2).

$$
\begin{gathered}
{\left[\begin{array}{c}
\dot{\mathrm{X}} \\
\dot{\mathrm{Y}} \\
\dot{\psi}
\end{array}\right]=\left[\begin{array}{ccc}
\cos \psi & -\sin \psi & 0 \\
\sin \psi & \cos \psi & 0 \\
0 & 0 & 1
\end{array}\right]\left[\begin{array}{c}
\dot{\mathrm{x}} \\
\dot{\mathrm{y}} \\
\dot{\psi}
\end{array}\right]} \\
{\left[\begin{array}{c}
\ddot{\mathrm{X}} \\
\ddot{\mathrm{Y}} \\
\ddot{\varphi}
\end{array}\right]=\left[\begin{array}{ccc}
\cos \psi & -\sin \psi & 0 \\
\sin \psi & \cos \psi & 0 \\
0 & 0 & 1
\end{array}\right]\left[\begin{array}{c}
\ddot{\mathrm{x}}-\dot{\mathrm{y}} \dot{\mathrm{y}}+\dot{\mathrm{x}} \dot{\psi} \\
\ddot{\psi}
\end{array}\right]}
\end{gathered}
$$

From the view of contact geometry, the wheel-rail shape is simplified to a cone to avoid massive nonlinear computation in which case both the slip forces and side forces are still considered in the model, as shown in Figure 6 (Masao, 2008). 


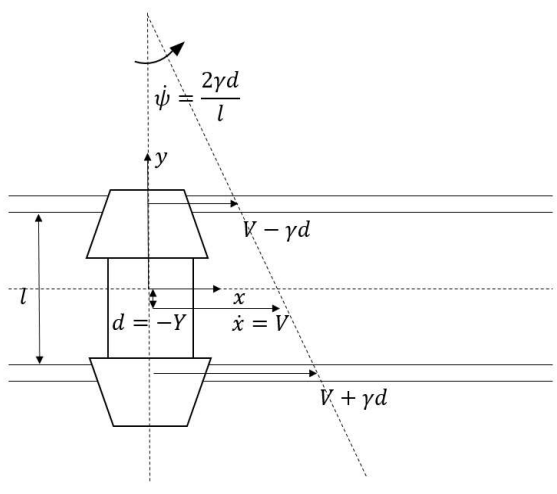

Figure 6. Wheels model.

The velocity in the moving coordinate system is given by:

$$
\left[\begin{array}{c}
\dot{\mathrm{x}} \\
\dot{\mathrm{y}} \\
\dot{\psi}
\end{array}\right]=\left[\begin{array}{c}
\mathrm{V} \\
0 \\
\frac{2 \gamma \mathrm{d}}{1}
\end{array}\right]
$$

Where $\gamma$ is the angle of the cone wheel, $\mathrm{d}$ is the distance between the center of the wheel and the center of the rail $(\mathrm{d}=-\mathrm{Y}), 1$ is the distance between wheels and $V$ is the speed in $x$-direction in local coordinates. When considering only the kinematics, as shown in equation (2) and (3):

$$
\ddot{\mathrm{Y}}=\mathrm{V} \cos \psi \dot{\psi} \approx \mathrm{V} \dot{\psi}=\frac{-2 \mathrm{~V} \gamma}{1} \mathrm{Y}
$$

This indicates that the assumption is able to model the hunting behavior of the lateral vibration of the wheelrail with the frequency of $\sqrt{2 \mathrm{~V} \gamma / 1}$.

Considering the adhesion and curve influence in the running direction, according to (Masao, 2008) the equation of the train dynamics in the moving coordinate system can be unified by equation (5):

$$
\begin{aligned}
& {\left[\begin{array}{ccc}
\mathrm{m} & 0 & 0 \\
0 & \mathrm{~m} & 0 \\
0 & 0 & \mathrm{I}
\end{array}\right]\left[\begin{array}{c}
\ddot{\mathrm{x}} \\
\ddot{\mathrm{y}} \\
\ddot{\psi}
\end{array}\right]+\frac{1}{\mathrm{~V}}\left[\begin{array}{ccc}
0 & 0 & 0 \\
0 & 2 \mathrm{c}_{22} & 0 \\
0 & 0 & \frac{1}{2} \mathrm{c}_{11}^{2}
\end{array}\right]\left[\begin{array}{l}
\dot{\mathrm{x}} \\
\dot{\mathrm{y}} \\
\dot{\psi}
\end{array}\right]} \\
& +\left[\begin{array}{ccc}
0 & 0 & 0 \\
0 & 0 & -2 \mathrm{c}_{22} \\
0 & 2 \mathrm{c}_{11} \frac{1 \gamma}{\mathrm{d}} & 0
\end{array}\right]\left[\begin{array}{l}
\mathrm{x} \\
\mathrm{y} \\
\psi
\end{array}\right]=\left[\begin{array}{c}
\mathrm{F}_{\mathrm{x}}-\mathrm{f} \\
\mathrm{F}_{\mathrm{y}}+\mathrm{m}\left(\frac{\mathrm{V}^{2}}{\mathrm{R}}-\frac{\mathrm{gh}}{1}\right) \\
\mathrm{M}_{\mathrm{z}}
\end{array}\right]
\end{aligned}
$$

Where $c_{11}$ and $c_{22}$ are the constants of Kaller contact theory, $\mathrm{h}$ is the height difference of outer side and inner side of the curves. $m$ is the equivalent mass obtained through the gravity calculation in the interface. $\mathrm{F}_{\mathrm{x}}, \mathrm{F}_{\mathrm{y}}, \mathrm{M}_{\mathrm{z}}$ are the friction forces and torques due to the adhesion which can be calculated by the slip rate. Assuming the slip rate is $\mathrm{s}$ and its component in $\mathrm{x}$ and $\mathrm{y}$ direction is $\mathrm{s}_{\mathrm{x}}, \mathrm{s}_{\mathrm{y}}$, with the self rotation $\psi$ :

$$
\left[\begin{array}{c}
\mathrm{F}_{\mathrm{x}} \\
\mathrm{F}_{\mathrm{y}} \\
\mathrm{M}_{\mathrm{z}}
\end{array}\right]=\mathrm{f} \cdot\left[\begin{array}{c}
\mathrm{s}_{\mathrm{x}} \\
\mathrm{s}_{\mathrm{y}} \\
\psi
\end{array}\right]
$$

With the radius of the wheel $\mathrm{R}$ and inertia $\mathrm{I}$, the relationship between slip rate $\mathrm{s}$ and the traction/braking forces is given by equation (7) and (8).

$$
\begin{gathered}
\mathrm{s}=\left\{\begin{array}{l}
\frac{\mathrm{R} \dot{\theta}-\mathrm{V}}{\mathrm{R} \dot{\theta}} \forall \text { Traction } \\
\frac{\mathrm{R} \dot{\theta}-\mathrm{V}}{\mathrm{V}} \forall \text { Brake }
\end{array}\right. \\
\text { R I } \ddot{\theta}=\mathrm{F}_{\text {traction }}+\mathrm{F}_{\text {brake }}
\end{gathered}
$$

The relationship between vehicle dynamics of the train and traction/braking forces can be obtained based on the equation (6). For even faster calculation, the library implements the simplification of (Polach, 2000) of equation (6). An approach assuming an ellipsoidal contact area is used, with semi-axes a, b and normal stress distribution according to Hertz to calculate the rail-wheel forces, as shown in Figure 7 (Polach, 2000).

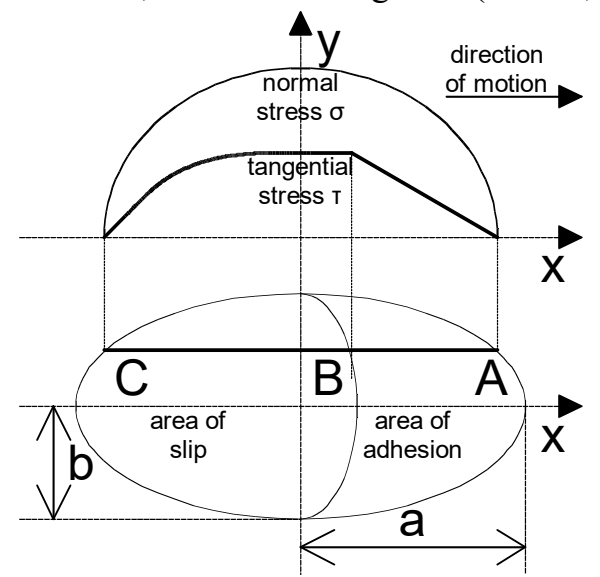

Figure 7. Assumption of the rail-wheel contact.

As a further simplification, a constant ellipse area is assumed. The maximum tangential stress $\tau$ is:

$$
\tau=\mathrm{f} \cdot \sigma
$$

where $\mathrm{f}$ is the coefficient of friction and $\sigma$ is the normal stress. $f$ is assumed constant in the contact area. The method assumes a linear displacement between the leading point $\mathrm{A}$ to the trailing point $\mathrm{C}$. At first, the contact area sticks firmly and the displacement is caused by the material creepage (area of adhesion). The tangential stress $\tau$ acts against the creep and grows linearly with the distance from the leading edge. If $\tau$ reaches the maximum value in the adhesion area, the relative movement of the contact area appears. This part is called area of slip.

Based on the theory, the relationship of the slip s and adhesion force is gotten. 


\subsubsection{Validation}

The wheel-rail contact model based on Modelica is built according to the theory mentioned in section 2.2.1. The parameters and coefficient of friction $f$ is assumed constant in the model as shown in Figure 8.

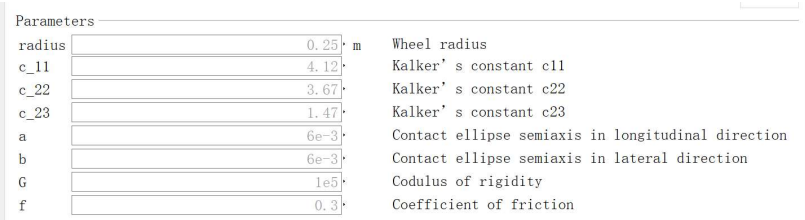

Figure 8. The parameters in the rail-wheel contact model. The model was validated against the data in (Polach, 2000). The model proposed in this paper uses the same theory but adds the framework to multi-body simulation. The bluish continuous trajectories of the creep displacements $s_{x}, x_{y}$ and spins $\psi$ in the first three sub-figures of Figure 9 were applied to the model. The set of red dots $0 . .9$ indicate the measurement values of (Polach, 2000). The other subfigures show the resulting creep forces. The plots demonstrate that the Modelica model is in congruency with the paper results and proofs that the model can be used for the contact calculation.
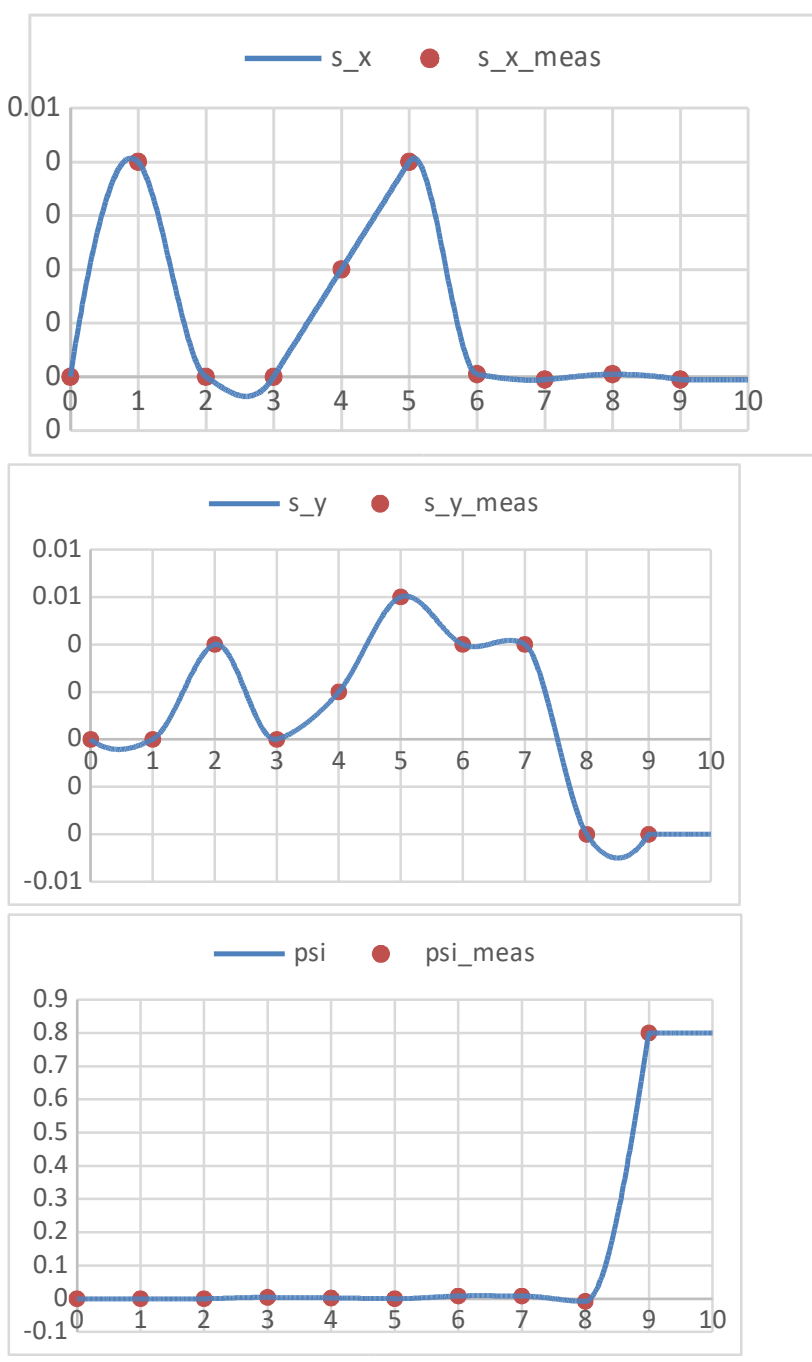
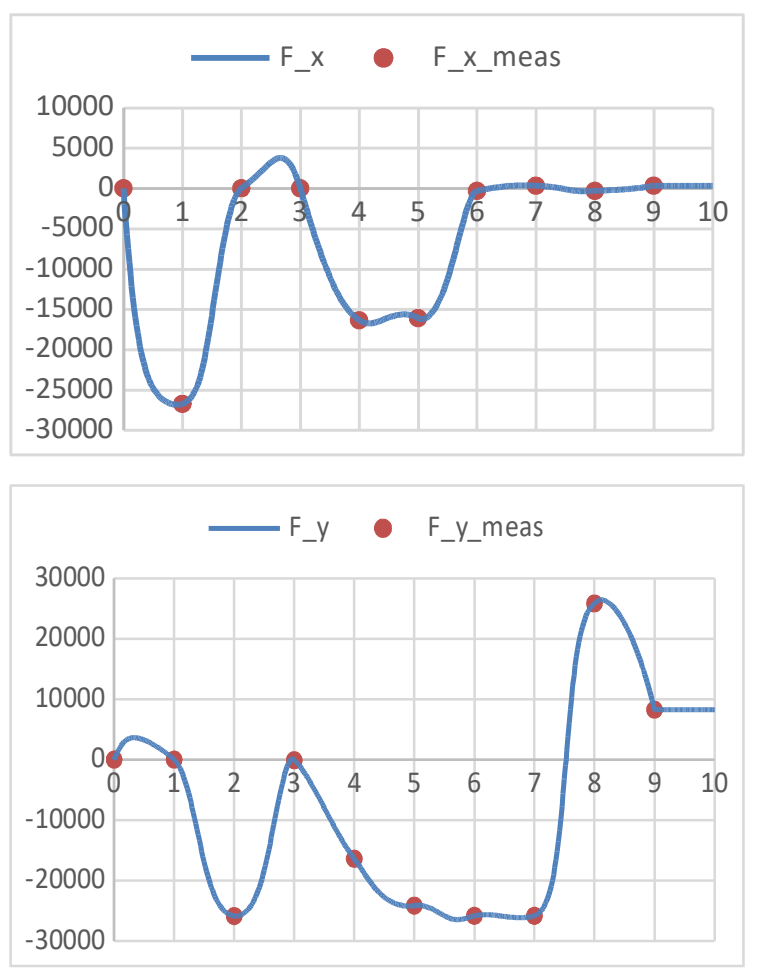

Figure 9: Validation of creep displacement and creep forces ( $x$-axis: data points (discrete)/time (continuous)).

\subsubsection{Outcome:}

The following tasks can be implemented based on the mechanical models:

- $\quad$ Simulate the threshold of the lateral hunting fluctuation under the irregular rail or external forces, and the stability when passing through the curves.

- Simulate the damping effect in vertical direction of the train for human comfort evaluation.

- $\quad$ Simulate the energy consumption of the integrated train along the rail.

\subsection{Thermal Fluid Modeling}

When estimating energy consumption of the train, besides the auxiliary power consumption such as lighting, two critical factors of thermal and water management (i.e., A/C, water supply and drainage system) should be considered.

The purpose of thermal management is to control the cabin temperature in a proper range for the passengers' comfort and control the temperature of traction system to ensure the normal operation. Water management is to ensure the water supply and drainage in the cabin and washroom.

Based on the purpose to evaluate the energy consumption of the integrated train and the model level the behavioral model of the thermal and fluid models were built without considering the complex shapes of the flow machinery and pipelines. Only the dynamic equivalent model was used to analyze the model behavior. 


\subsubsection{Implementation}

The main cooling types of the thermal management of the trains are air cooling and liquid plus air cooling. Compared to the thermal management system of automobiles, the biggest difference is that with the increase of the running speed, the demanded pressure difference increases, the flow decreases which may lead to the equipment being broken due to overheat in severe conditions. So it is critical to control the air conditioning system and cooling system according to the running speed.

The traction converter is liquid cooled, where the model consists of 4 cold plates, a pump and a cooling tank. The cooling medium is ethanol plus water.

The traction transformer is cooled through forced air cooling mixed with oil cooling. The cooling unit circulates the oil from the transformer to the oil cooling device by a pump where the heat is scattered by a fan.

The traction motors are cooled by forced air with one air inlet and two air exhausts whose air volume should coincide as good as possible.

The HVAC system of the train is mainly based on ventilation, which does not involve two phase flow. It provides cold air in summer and warm air in winter, as shown in Figure 10.

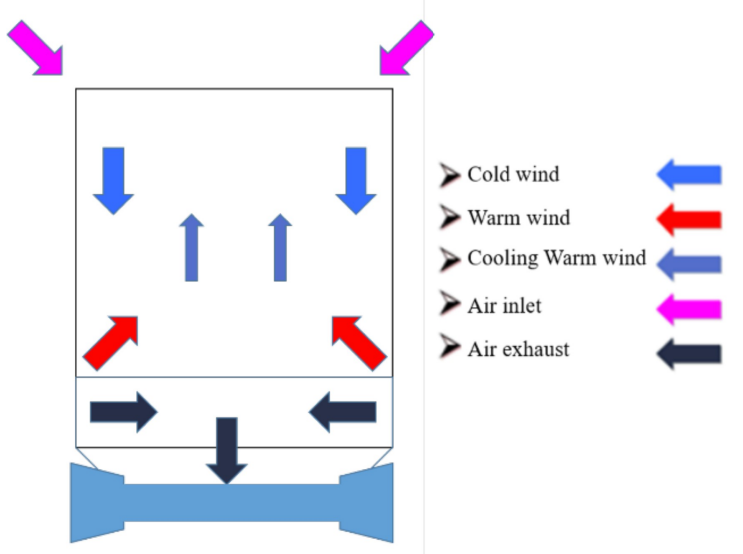

Figure 10. HVAC System in the train.

Water supply is added by further models where the dynamic behavior of the car may be influenced by the mass distribution.

\subsubsection{Demonstration}

CoolingLib was used to design cooling units, such as converter cooling unit, transformer cooling unit, and motor cooling unit. Figures 11 to 13 show the models and validation results (textual): the medium of the traction converters is water-glycol mixture solution, the medium of the transformer is $45 \#$ transformer oil.

Passenger comfort was evaluated by the HumanComfortLib by XRG Simulation GmbH. The

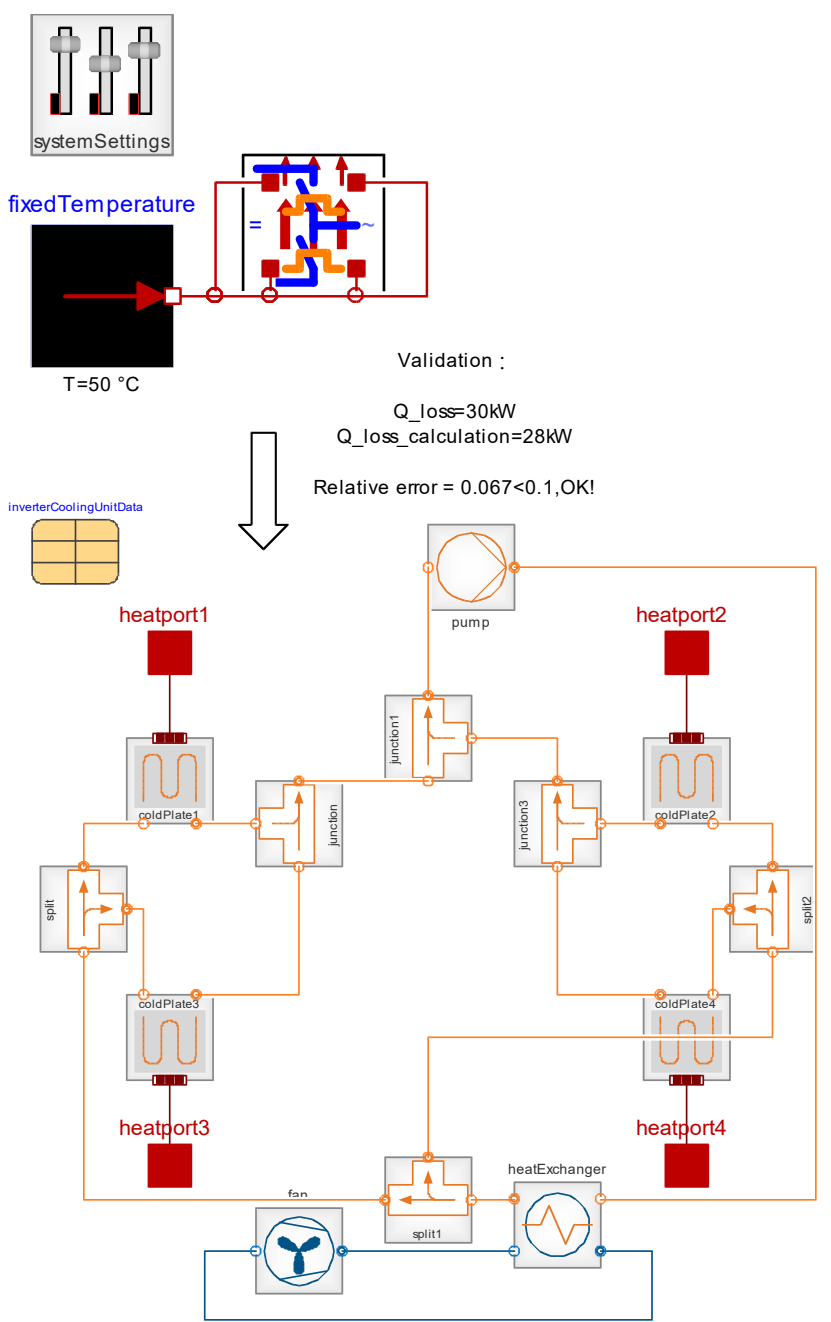

Figure 11. Traction converter cooling unit and Validation.

air conditioning and water supply system models were validated according to the passenger flow.

\subsubsection{Outcome:}

The thermal fluid model can be used to implement the following work:

- Simulate the cooling effect of the traction system and calculate the thermal capacity of the traction system.

- Simulate the air conditioning effect of the train for the human comfort evaluation

- Simulate the energy consumption of the auxiliary system in air conditioning system and water supply and drainage system to evaluate the energy consumption of the integrated train. 


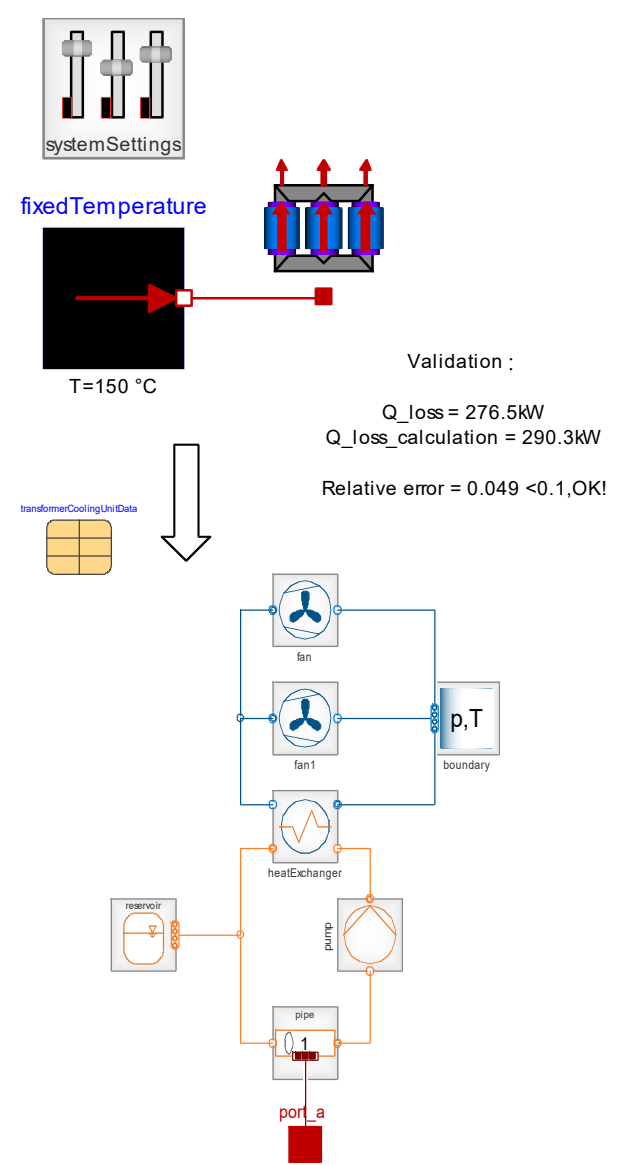

Figure 12. Transformer cooling unit and Validation.

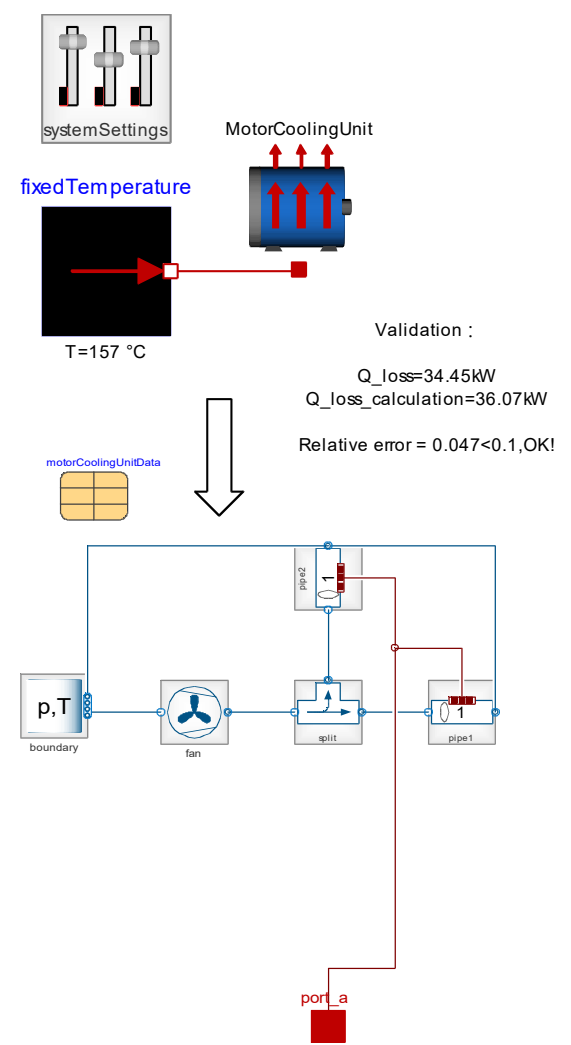

Figure 13. Motor cooling unit and Validation.

\subsection{Traction System and Control Logics}

The traction control and the control logics are closely linked and can be found in the figures presented in this chapter. The train control logic is of enormous relevance for industrial application and at the same time of limited scientific interest. For the sake of completeness, however, it should also be mentioned here.

\subsubsection{Overview}

Figure 14 shows an overview of the EMU structure. Each train consists of 8 cars where the rear 4 cars are mirror-inverted identical to the front part. Two trains can be coupled to a total number of 16 cars.

In nominal operation each half of a train is electrically independent and draws the power via the pantograph (T03/T06) from the power supply line. The circuit is grounded via the wheels and the railway. The power is transformed in the car with the pantograph to a lower voltage which is fed to the neighboring cars (M02/M04, M05/M07). Only those are driven cars with motors where the power is converted in these cars by traction current converters. The cars with the train conductor's cabins contain the auxiliary current converters and batteries and battery chargers.
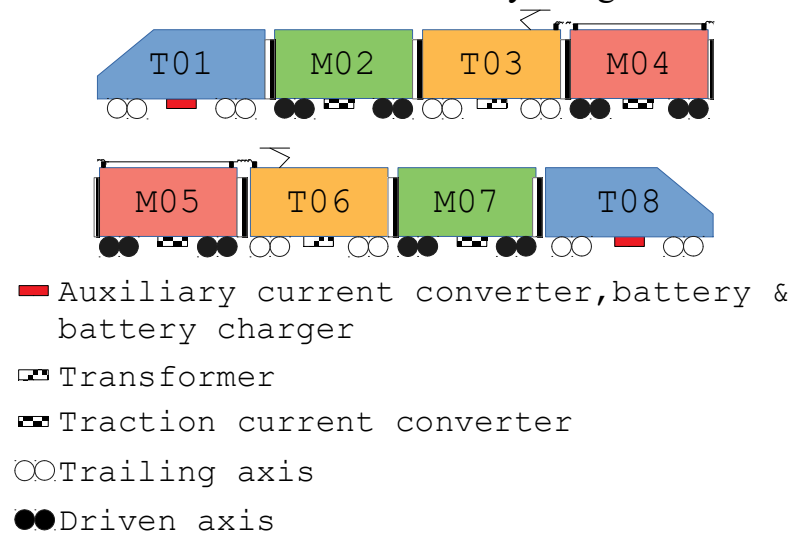

Figure 14. Schematic diagram of traction system.

\subsubsection{Implementation}

The electrical system is shown in Figure 18 (some elements are hidden for better clarity). The model shows the electrical system of the train with cars 1-8. Mechanics are simulated by the element (9). The train is fed by the catenary. The impedance of the line and the railway (1) has substantial effects on the power quality to the line and maximum power off-take from the line. Considerations on the impedances can be found in (Hill et al., 1989; Allenbach, 2014). For the Chinese High Speed Railway the following parameters were assumed from literature:

\begin{tabular}{|c|c|}
\hline Source voltage: & $25 \mathrm{kV}$ \\
\hline Net frequency: & $50 \mathrm{~Hz}$ \\
\hline Series Resistance : & $0.09 \Omega / \mathrm{m}$ \\
\hline
\end{tabular}




\begin{tabular}{|c|c|}
\hline Series Inductance: & $0.669 \mu \mathrm{H} / \mathrm{m}$ \\
\hline Parallel Capacitance & $7 \mathrm{nF} / \mathrm{m}$ \\
\hline Parallel Conductance & $18 \mu \mathrm{S} / \mathrm{m}$ \\
\hline $\begin{array}{c}\text { Typical distance to } \\
\text { power station }\end{array}$ & $14.7 \mathrm{~km}$ \\
\hline
\end{tabular}

Modelling does not take into account a dynamic change of the length but is implemented by Modelica.Electrical.Analog.Lines.Oline.

While the pantograph (2) is one of the most complex mechanical elements due to the high-speed operation, the Modelica model is limited to a resistance and sensors for net voltage and transferred current.

The voltage is transformed to a lower voltage level of around 2000 Volts by the transformers (4). The correct estimation of losses and harmonics demands a simulation with magnetic hysteresis effect. The Modelica.Magnetic library (Ziske and Bödrich, 2012) offers accurate and fast methods while the choice of magnetic core material is very limited. The transformer parameterization is crucial for the correct estimation of harmonics. Parameters may come from detailed design or hardware tests.

Number (3) is the power conversion system including $\mathrm{AC}$ to $\mathrm{DC}$ bi-directional converters, intermediate voltage circuit, current converter and traction motors.

(5) to (9) are control logics: (5) communication models , e.g. passing neutral phase information, (6) the driver's commands, (7) the Central Control Unit of the train, (8) the Traction Control Unit with one TCU per driven car.

The traction system is shown in detail in Figure 19. For this train, the AC input voltage (left) is converted into an $\mathrm{DC}$ intermediate voltage and inverted to $\mathrm{AC}$ voltage by (20) to feed the parallel traction motors. The input converter should be able to convert the $\mathrm{AC}$ voltage passively into a lower DC voltage if no traction but only auxiliary power is needed, boost the AC voltage to a higher intermediate DC level or feed regenerated power back to the network when braking. This fourquadrant converter needs to be Power Factor Correcting (PFC) for good power quality in the supply line without interaction of trains, losses by reactive power or radiated harmonic noise.

Figure 15 shows two typical converter topologies. The converter with Neutral Point Clamped (NPC) has the benefit that all transistors only have to resist to half the DC voltage. In the last years new IGBTs were developed which are rated for the higher voltage level which enables the second type of converter: the interleaved boost converter. For both converters, transformer leakage inductance can be used as the boost inductances $L_{1} / L_{2}$ respectively $L_{3}$ to $L_{6}$. Due to the redundancy and other benefits, the interleaved converter was implemented in the library (Figure 19:
11,12, with precharge 10 and control 13). Considerations on the efficiency and rating of both topologies can be found in (Bellini et al., 2002) and (Musavi et al., 2010).

Typically the switching frequency is selected low to prevent switching losses. If the switching frequency is below 11 times the natural time constant of the input impedance, then simple current controls may fail (Freyberger, 2002). The library offers an advanced control concept, including a reliable phase detection and control in dq system.

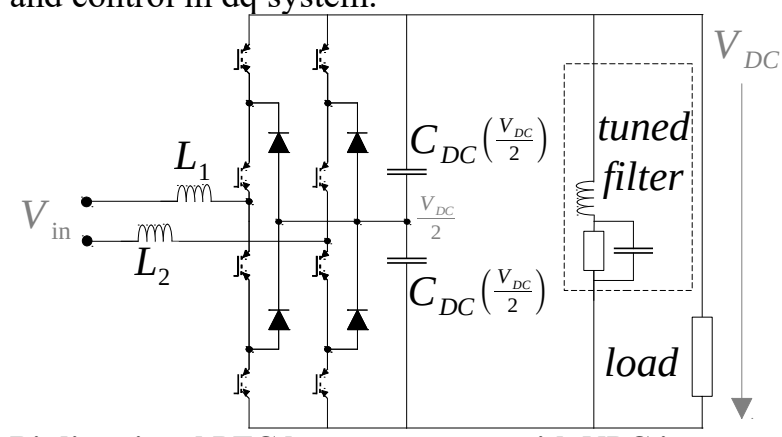

Bi-directional PFC boost converter with NPC inverter

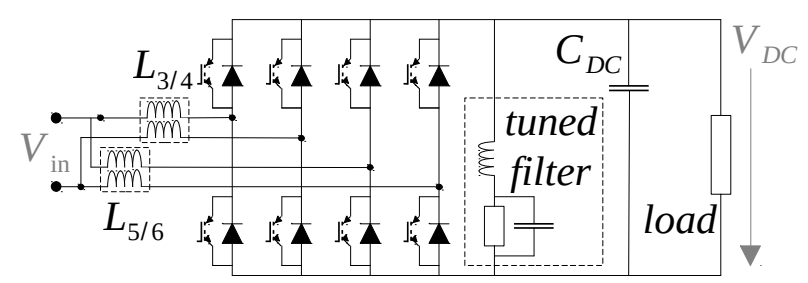

Bi-directional PFC interleaved boost converter

Figure 15. Boost converter topologies.

For both types of boost converters, the control aims to preserve power quality on the AC side $V_{\text {in }}$ by drawing power from $V_{D C}$ at double net frequency. The ripple can be attenuated by a tuned filter (14) of first or second order. The intermediate voltage is stabilized by capacitors (15). Power is drawn from the auxiliary system (17) or the traction inverter (19). Each traction converter feeds four induction motors connected in parallel. (16) and (18) are overvoltage protection and braking resistor.

\subsubsection{Demonstration}

The electrical model is demonstrated by a study of the net side power quality. The input current of one transformer feeding two conversion/traction units shall be investigated at nominal load (e.g. car 2-4). For better efficiency, the traction system is simulated by a more simple controlled power load.

Results are shown in Figures 16 and 17. The former one shows the currents on the transformer's secondary windings which are disturbed by pulse-widthmodulation (PWM), where the currents combine to a smooth current in the primary windings. Figure 17 shows the load profile and transient of the actively 

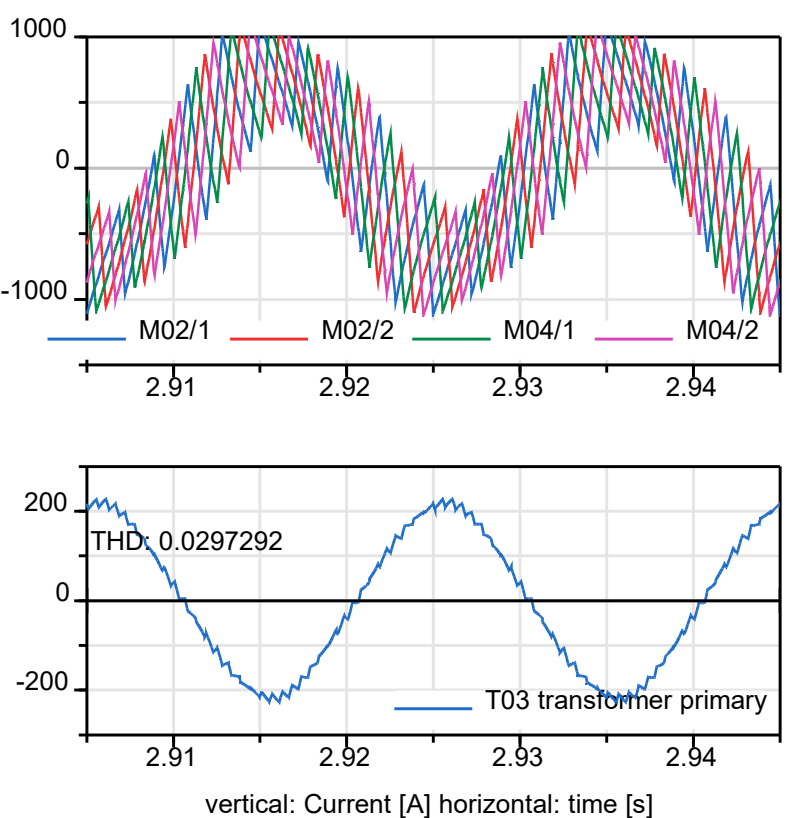

Figure 16. Harmonic test of current drawn by one transformer. Top: Currents of each interleaved converter. Bottom: transformer input current and THD.

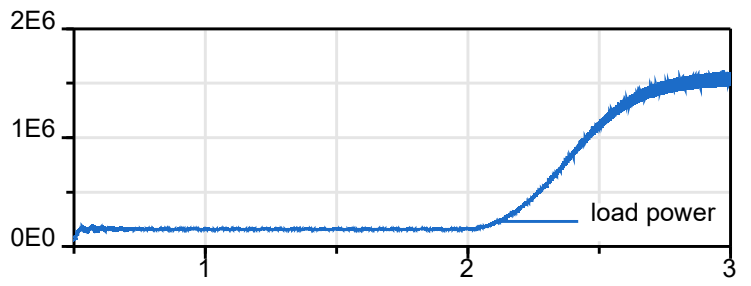

vertical: Power [W] horizontal: time [s]
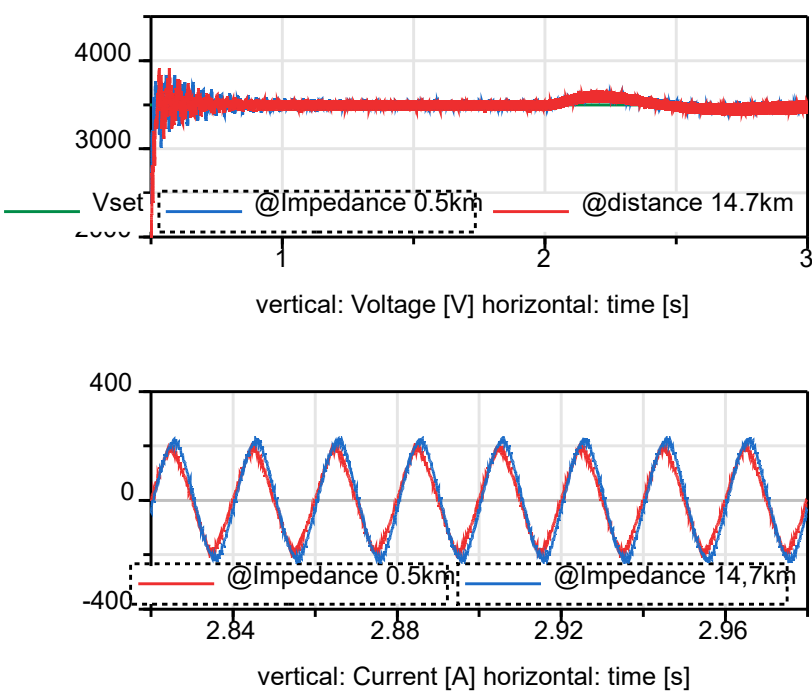

Figure 17. Harmonic test of current drawn by one transformer. Top: Load profile. Middle: Trajectory of controlled DC intermediate voltage @ distance $0.5 \mathrm{~km}$ and $14.7 \mathrm{~km}$ to power source. Bottom: Current shape for distance $0.5 \mathrm{~km}$ and $14.7 \mathrm{~km}$.

controlled DC intermediate voltage and $\mathrm{AC}$ input currents at two impedance values reflecting $14.7 \mathrm{~km}$ and $0.5 \mathrm{~km}$ to the power station. The THD is well below the limit of the industrial standard.

\subsubsection{Outcome:}

All tasks which are listed above rely on the electrical models and control models. Detailed electrical models are utilized for

- sizing of electrical components for stability, power quality and rating, like inverter bridges, transformers, passive components,

- detailed sizing of heat dissipation in connection with simplified cooling system,

- validation of simplified models.

Simplified non-fast-switching models are used for

- Vehicle energy consumption estimation in conjunction with simple vehicle dynamics and power systems

- Traction system thermal capacity estimation for cooling system layout and control of power reduction

\section{Conclusion and future work}

In this paper, needs and implementations for system modeling of high speed trains with focus on the Beijing-Zhangjiakou Intercity Railway were shown. The models cover all relevant domains and tests and optimization for the real train system will be performed with it in the near future.

\section{Acknowledgment}

The authors gratefully acknowledge ZEMT for their help of providing the system information and test data. SIMTEK also cooperated with ZEMT on the control and health monitoring of the braking system.

\section{Bibliography}

Allenbach, J.-M. (2014). Eisenbahntechnik (German). Available

https://documents.epfl.ch/groups/t/tr/traction/www/docum ents/ZusammET.pdf.

Bellini, A., Bifaretti, S., and Constantini, S. (2002). High power factor converters for single-phase AC traction drives. In WIT Transactions on The Built Environment 61.

Belmon, L., and Liu, C. (2011). High-speed train pneumatic braking system with wheel-slide protection device: A modelling application from system design to HIL testing. In Proceedings of the 8th International Modelica Conference; March 20th-22nd; Technical Univeristy; Dresden; Germany, p. 549-556.

Dumont, E., and Maurer, W. (2012). DyMoRail: A Modelica Library for modelling railway buffers. In Proceedings of the 9th International MODELICA Conference; September 3-5; 2012; Munich; Germany, p. 691-696. 
Franke, R., and Wiesmann, H. (2014). Flexible modeling of electrical power systems--the Modelica PowerSystems library. In Proceedings of the 10 th International Modelica Conference; March 10-12; 2014; Lund; Sweden, p. 515-522.

Freyberger, F. (2002). Leittechnik: Grundlagen, Komponenten, Systeme; Projektierung, Steuerung und Regelung, Signal-, Bussysteme, Aktoren, Sensoren (German). Pflaum.

Frilli, A., Meli, E., Nocciolini, D., Pugi, L., and Rindi, A. (2016). Energetic optimization of regenerative braking for high speed railway systems. In Energy Conversion and Management 129, p. 200-215.

Heckmann, A., Keck, A., Kaiser, I., and Kurzeck, B. (2014). The foundation of the dlr railwaydynamics library: the wheel-rail-contact. In Proceedings of the 10 th International Modelica Conference; March 10-12; 2014; Lund; Sweden, p. 465-475.

Hill, R., Carpenter, D., and Tasar, T. (1989). Railway track admittance, earth-leakage effects and track circuit operation. In Railroad Conference, 1989. Proceedings.,

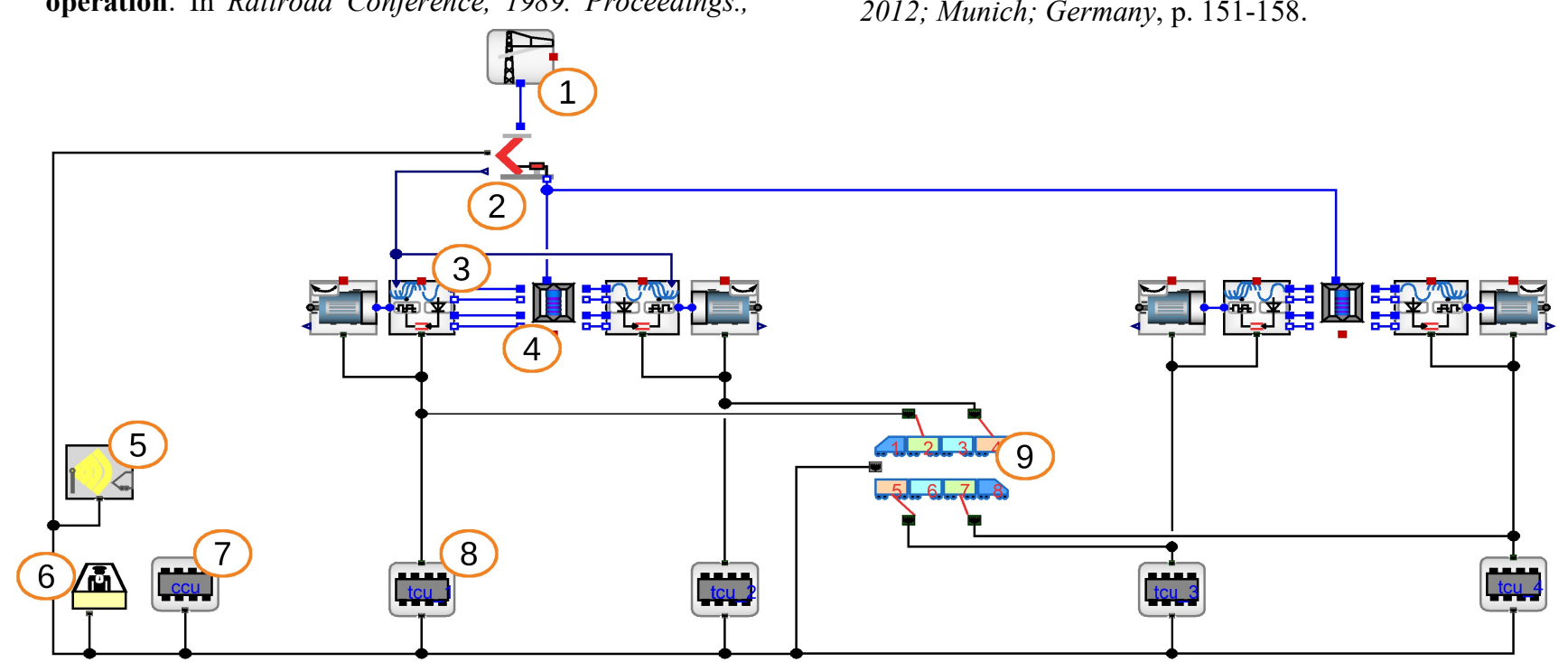

Figure 18. Modelica diagram of test model of the traction system (simplified).

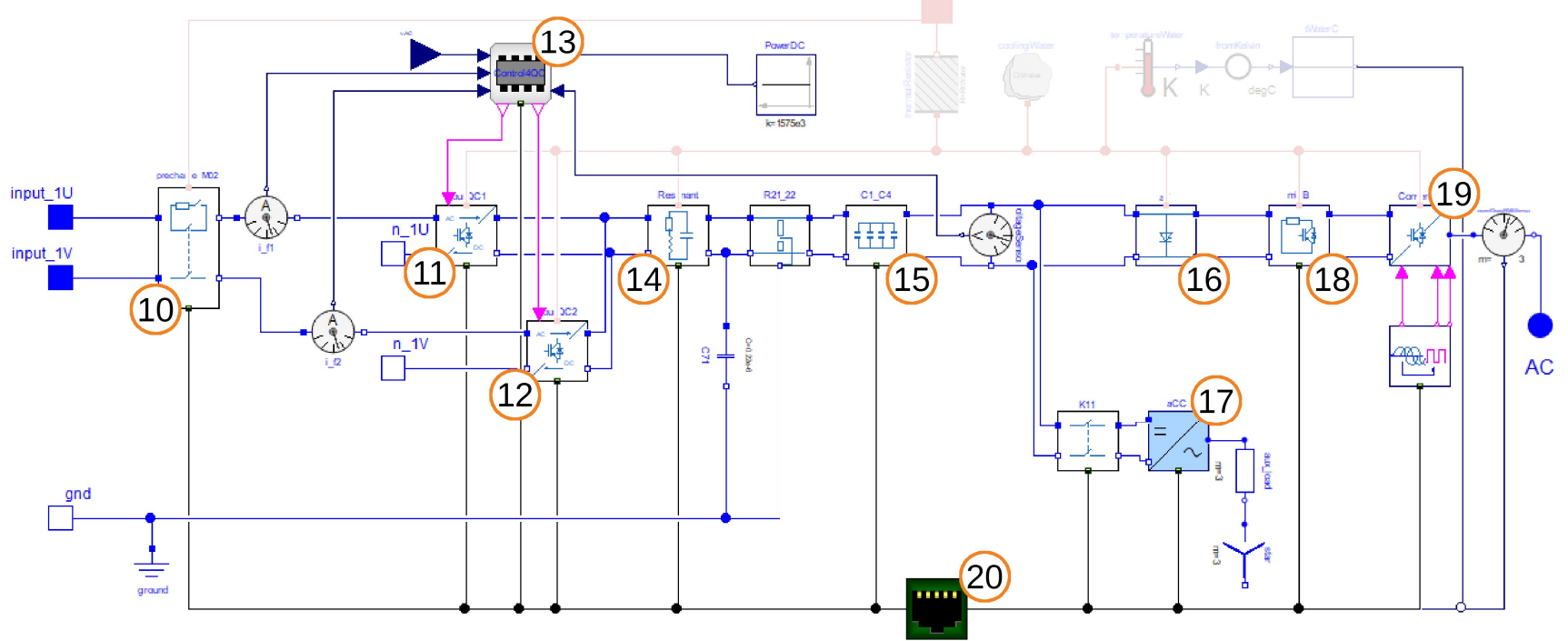

Figure 19. Schematic diagram of the power conversion system. 